Check for updates

Cite this: RSC Adv., 2018, 8, 1124

\title{
Fading and showing mechanisms of ancient color relics based on light scattering induced by particles
}

\author{
L. Z. Zheng, X. T. Liang, S. R. Li, Y. H. Li and D. D. Hu (D)*
}

Fading and showing mechanisms of ancient color paintings based on light scattering induced by particles were proposed. To confirm the mechanisms, simulated and application experiments were carried out to restore an ancient blurred color painting. Loading $\mathrm{TiO}_{2}$ particles $(500-1000 \mathrm{~nm}$ ) onto a piece of colored paper could result in blurring of the color of the paper, which is attributed to light scattering caused by air voids between the particles. Filling air voids with ionic liquid (a non-volatile solution with a high refractive index) could highlight the color by reducing scattering. These results were experimentally testified by the combination of a fluorescence probe and multi-angle reflectance spectra, in which scattering decreased the incident optical path in the painting layer while the incident optical path was increased by filling the air voids with ionic liquid. As a practical example, the proposed method was applied to highlight an ancient Chinese painting with blurred color. This investigation is very useful to restore faded color paintings.

Received 30th October 2017 Accepted 15th December 2017

DOI: 10.1039/c7ra11923b

rsc.li/rsc-advances time goes by and thereby the color is degraded. In addition, surface properties pertaining to roughness might also cause color changes.

Actually, the evolution of color is the result of complex physical and chemical reaction of a pigment. In general, the color is affected not only by material components but also the surface microstructure. A color affected by material components is often noticed. The change in color caused by the variation of the pigment is attributed to this. However, a color affected by the surface microstructure is usually neglected. One of the important factors is that the color is affected by scattering caused by the surface microstructure. In principle, the scattering of light depends on the size of the dispersed phase as well as the relative refractive indices of the dispersed phase and the continuous phase. If the size of the dispersed phases is approximately equal to the wavelength of light, Mie scattering predominates. Mie scattering is not strongly wavelengthdependent and scatters all visible light. This means that for a painting layer containing this kind of dispersed phase, the color of the painting layer is consequently whitened.

A large number of studies have shown that most pigments used in Chinese murals and painted sculptures are natural minerals. Natural mineral pigments are stable under normal conditions. ${ }^{4}$ From this point of view, the fading of color in ancient Chinese paintings is not mainly related to the change of pigment. According to the principle of the surface microstructure affecting the color, it can be assumed that the fading of ancient Chinese paintings is probably related to light scattering.

In ancient Chinese color paintings, the painting layer contains pigment particles and a binder. The binders are usually organic materials such as egg and animal glue..$^{5,6}$ These
Engineering Research Center of Historical and Cultural Heritage Protection, Ministry of Education, School of Materials Science and Engineering, Shaanxi Normal University, Xi'an 710062, China. E-mail: daodaohu@snnu.edu.cn; Tel: +86-029-81530717 
organic materials in the painting layer can easily be deteriorated and this leads to the appearance of a lot of pores in the painting layer. In this case, the difference in the refractive index between the pigment particles and the binding medium is increased due to the presence of air voids in the painting layer. As a result, the amount of light scattering in the painting layer is increased, which decreases the intensity of light absorbed by the pigment, leading to the lightened color of the pigments. Based on the above-mentioned principle, it is a very popular idea that scattering can cause color changes in ancient Chinese color paintings.

Motivated by the change of color caused by scattering and the interest in showing the faded original appearance, in this paper we analyze a collected color painting (Yuan Dynasty, 1316, Hancheng Puzhao Temple, Shaanxi, China) and carry out an experiment on the mechanism of discoloration and visualization based on light scattering induced by $\mathrm{TiO}_{2}$ nanoparticles. A faded paper sample was prepared by loading $\mathrm{TiO}_{2}$ particles on colored paper to enhance scattering (Fig. 1a). To show the faded color, a trace amount of ionic liquid was introduced into the faded paper to decrease the scattering caused by $\mathrm{TiO}_{2}$ particles (Fig. 1b). Both the simulated experiment and practical application confirmed that the decrease of scattering strengthens the incident light to restore the color of a faded painting. To the best of our knowledge, this is the first example to reduce scattering for the restoration of faded heritage relics.

\section{Experimental}

\subsection{Reagents and materials}

Tetrabutyl titanate (TBOT, analytical grade, Kemiou Chemical Reagent Co., Ltd, Tianjin, China), 1-butyl-3-methylimidazolium

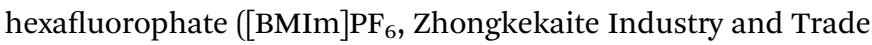
Co., Ltd, Lanzhou, China), ethanol, and acetone (analytical

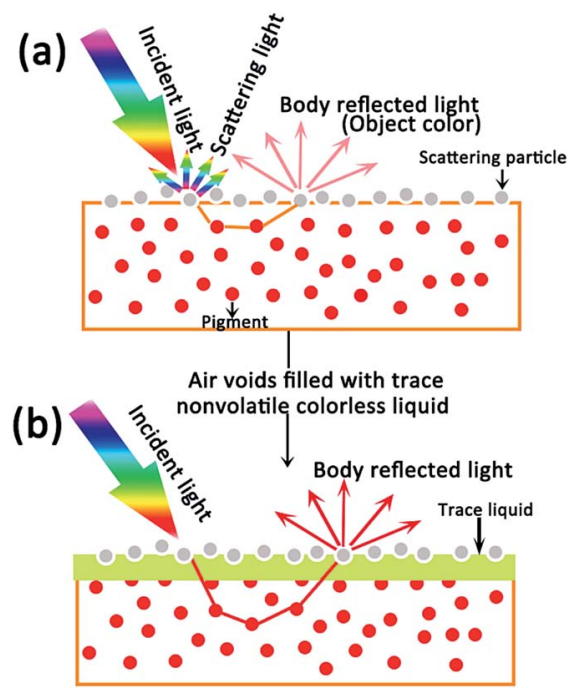

Fig. 1 Schematic illustration of scattering induced by particles and the elimination of scattering by filling the voids. grade, Sinopharm Chemical Reagent Co., Ltd, Shanghai, China) were used without further purification.

Red napkin paper and drinking paper were purchased from a local supermarket. Red napkin paper was used as the colored matrix to load $\mathrm{TiO}_{2}$ nanoparticles for the construction of a model sample with light scattering. In principle, any colored paper with abundant pores could be used for this purpose. Drinking paper is used to construct a model sample with adjustable scattering thickness through compressing multilayer drinking paper. In principle, here any colorless drinking paper (absorbent paper) with abundant pores could be selected.

\subsection{Fabrication of the light scattering paper sample}

2.2.1. Fabrication of single-layer paper with enhanced light scattering. A piece of red napkin paper (RNP) was firstly dipped into the TBOT alcohol solution (20\% volume ratio of TBOT to alcohol) in a glass dish. Then, the immersed RNP was placed on a mesh in a closed container containing water at the bottom, and the container was placed in an oven at $30^{\circ} \mathrm{C}$. After 2 hours, RNP was dried at room temperature, and RNP loaded with $\mathrm{TiO}_{2}$ nanoparticles was obtained. The final sample was named $\mathrm{S}$ RNP. Due to S-RNP being loaded with $\mathrm{TiO}_{2}$ particles, the scattering of S-RNP is stronger than that of RNP.

2.2.2. Fabrication of a sample with multi-layer scattering. To explore the effect of light scattering on the depth of the incident light entering a sample, a sample with adjustable scattering thickness was prepared. To this end, 12 stacked sheets of drinking paper (DP) were compressed using a pressure machine. This sample was named MLS-DP. The scattering thickness could be adjusted by peeling off paper from MLS-DP.

\subsection{Showing the color of the simulated faded paper}

To show the color of faded paper caused by the scattering of air voids, a trace amount of liquid was used to fill the air voids in the faded paper to show color through decreasing the scattering. In this investigation, the liquid used for showing the color of simulated faded paper was a mixture of ionic liquid ([BMIm] $\left.\mathrm{PF}_{6}\right)$ and acetone, and S-RNP was used as the simulated faded paper. Typically, the volume ratio of the mixture was 0.5 for $[\mathrm{BMIm}] \mathrm{PF}_{6}$ to acetone. The S-RNP sample was coated with a suitable amount of the mixture. After the evaporation of acetone, the ionic liquid remained in the S-RNP sample, showing the color of the simulated faded paper.

\subsection{Showing the color of the faded ancient painting}

Following the above-mentioned process, the faded painting (Yuan Dynasty, 1316, Hancheng Puzhao Temple, Shaanxi, China) was treated by a similar manner to restore color.

\subsection{Characterization}

2.5.1. Surface morphology and composition. The morphology and chemical composition of the samples were characterized by scanning electron microscopy (SEM, Quanta 200, FEI, Netherlands) equipped with an energy dispersive X-ray spectrometer (EDS). All samples were coated with gold prior to 
testing and the accelerating voltage was set as $20 \mathrm{kV}$ in the high vacuum mode.

2.5.2. Multi-angle reflectance spectroscopy. According to the Commission Internationale de L'Eclairage (CIE) LAB color scale, the color differences of the samples were measured and the reflectance spectra were recorded in the range of 400$700 \mathrm{~nm}$ using a multi-angle spectrophotometer (MA98, X-Rite, USA) with the colorimetric illuminant of D65. The MA 98 multi-angle spectrophotometer could provide a color image, and the $L^{*}, a^{*}$, and $b^{*}$ values and the reflectance spectra of the samples obtained from various viewing directions. The measurement angles and illumination angles are shown in Fig. 2. In actual measurement, each of these experiments was repeated at least three times, and representative results are given. Standard deviations were below $5 \%$, and this deviation would not affect our conclusion.

2.5.3. Characterization of the optical path in scattering paper. To explore the effect of scattering on the optical path of the incident light in the sample, a new method was established based on fluorescence spectroscopy to probe the optical path. Printing paper with fluorescence used as a probe was placed under the MLS-DP sample, and the fluorescence spectra were recorded in the case of MLS-DP with different numbers of layers through peeling off the paper from MLS-DP. The change in the fluorescence intensity could reflect the depth of incident light entering the multi-layer paper. The fluorescence spectra were recorded on a fluorescence spectrophotometer (F-7000, Shimadzu, Japan) with an excitation wavelength of $320 \mathrm{~nm}$. In actual measurement, each of these experiments was repeated at least three times, and standard deviations were less than $5 \%$, and this deviation would not affect our conclusion.

\section{Results and discussion}

3.1. Effect of loading particles on the morphology and color of paper

3.1.1. Morphology of the paper loaded with $\mathrm{TiO}_{2}$. According to the preparation process, the TBOT in RNP was hydrolyzed in situ to form $\mathrm{TiO}_{2}$ particles as the paper was placed in a humid environment. As mentioned above, the sample, RNP loaded with $\mathrm{TiO}_{2}$ particles, named S-RNP, was obtained. SEM images of RNP and S-RNP are shown in Fig. 3. As observed in Fig. 3a, RNP is composed of crossing fibers without obvious particles, while in the SEM image of S-RNP shown in Fig. 1b, a lot of particles

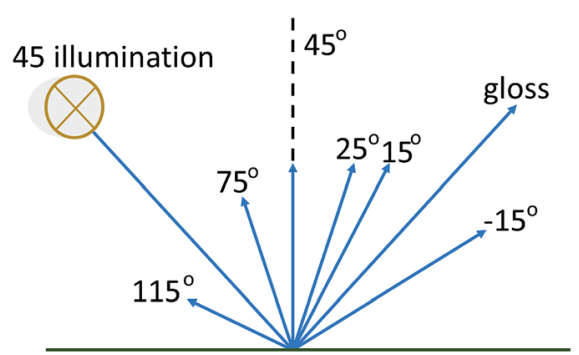

Fig. 2 Sketch of medium illumination and in-plane detection with MA98.
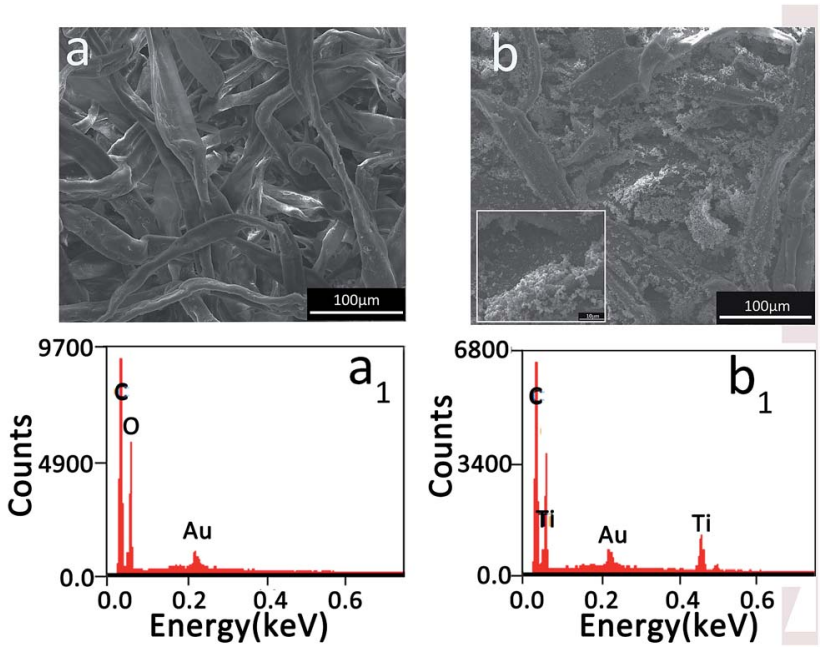

Fig. 3 SEM images and EDS spectra of the red napkin paper sample (a and $\left.a_{1}\right)$ and the S-RNP sample $\left(b\right.$ and $\left.b_{1}\right)$. The inset is the high magnification SEM image of part of (b).

appear in the range from 500 to $1000 \mathrm{~nm}$ (Fig. 3b, inset). Obviously, S-RNP with nanoparticles was successfully prepared. The EDS spectra of the RNP and S-RNP samples are shown in Fig. $3 a_{1}$ and $b_{1}$. Ti is found in the spectrum of the S-RNP sample but not in that of RNP. These results confirm that $\mathrm{TiO}_{2}$ particles were loaded on RNP. As a result, abundant air voids between the particles formed.

3.1.2. Color of paper loaded with $\mathbf{T i O}_{2}$. The reflectance spectrum reveals information about the hue, lightness, and chroma ${ }^{7}$ of color and is widely applied to the characterization of color. ${ }^{8-11}$ To investigate the effect of light scattering caused by particles on the color, the reflectance spectra of RNP and S-RNP were measured. Fig. 4 shows the reflectance spectra, first
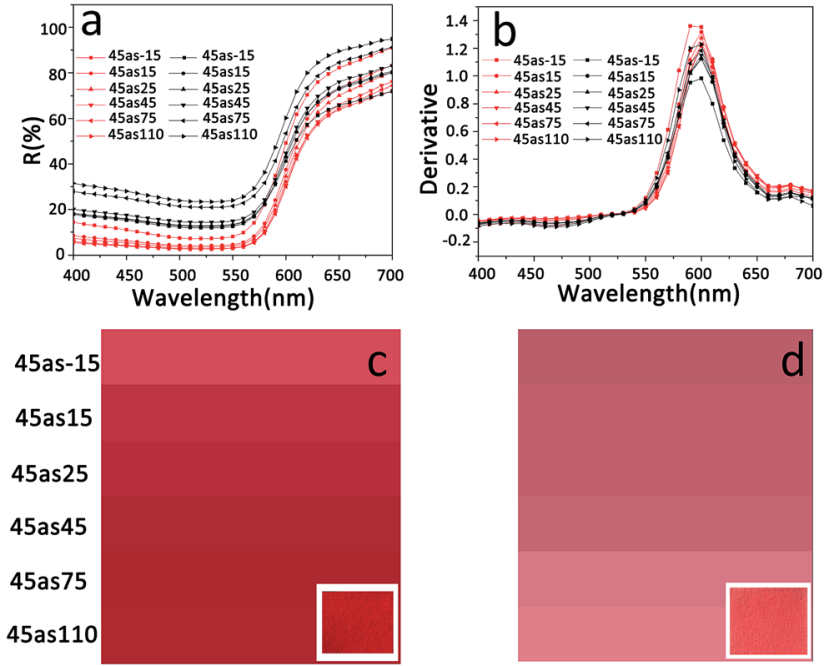

Fig. 4 (a) Multi-angle reflectance spectra (black curves: S-RNP, red curves: red napkin paper); (b) first derivative spectra (black curves: SRNP, red curves: red napkin paper); (c) and (d) color images in different viewing directions (left: red napkin paper, right: S-RNP). The insets in (c) and (d) refer to photos of the corresponding samples. 
derivative spectra, and color images of RNP and S-RNP. In Fig. 4a, similar to those of RNP, the reflectance spectra of S-RNP show an "S" shape, indicating the same hue of both samples. Additionally, as shown in Fig. 4b, in the first derivative spectra of RNP and S-RNP, a peak at about $600 \mathrm{~nm}$ appears, indicating that the loading of $\mathrm{TiO}_{2}$ particles did not change the red hue of the paper. It is worth mentioning that S-RNP has a higher reflectance value (as shown in Fig. 4a) in comparison to RNP, which implies that less incident light was absorbed by the dye in S-RNP and the specific color of S-RNP was blurred. In fact, this difference between RNP and S-RNP is obviously shown in the corresponding color images (Fig. 4c and d). The lightest color appeared at the propagation direction of the incident light for RNP while it appeared at the incident direction for S-RNP.

In order to further certify the above analysis, the $L^{*}, a^{*}$, and $b^{*}$ values for RNP and S-RNP were measured and are listed in Table 1 . The $L^{*}$ value of S-RNP is higher than that of RNP, indicating that S-RNP has a stronger reflectivity in comparison to RNP. In addition, both the $a^{*}$ and $b^{*}$ values of S-RNP are obviously smaller than those of RNP. According to the equation for chroma, ${ }^{21} C^{*}=\left(a^{*}+b^{*}\right)^{1 / 2}, C^{*}$ for RNP and S-RNP is 62.94 and 41.72, respectively, indicating that S-RNP loaded with $\mathrm{TiO}_{2}$ particles presents a lower chroma than RNP. The hue angles of RNP and S-RNP are 28.68 and 20.54, respectively, indicating that they have the same hue in the color wheel. ${ }^{12,13}$ In other words, the particles loaded on the paper did not influence the hue but caused the color to fade.

As is known, the light of many natural rough surfaces is a mixture of specular reflection and diffuse reflection. ${ }^{\mathbf{1 4 - 1 6}}$ The reflectance envelopes generated from Fig. 4 are shown in Fig. 5. The intensities (red curve) at all angles for the RNP surface are obviously higher than those of S-RNP (black curve), which indicates that the light absorption ability of RNP is stronger than that of S-RNP. Additionally, the RNP surface has the reflection characteristics of a natural rough surface due to the relatively high light intensity distributed in the reflective direction. ${ }^{17}$ However, for the S-RNP surface, the relatively high diffuse intensity is located at the incident direction (the black curve in Fig. 5). This feature is in agreement with the property of Mie scattering. ${ }^{18}$ Obviously, this feature is related to the scattering from the $\mathrm{TiO}_{2}$ nanoparticles in the S-RNP sample as observed in Fig. 3.

All the above results can be explained. Obviously, the difference in color between RNP and S-RNP is attributed to scattering. It is well known that the scattering efficiency is governed by a few factors. Firstly, scattering is strongly

Table 1 CIE $L^{*}, a^{*}$, and $b^{*}$ values of the samples ${ }^{a}$

\begin{tabular}{llll}
\hline Sample & $L^{*}$ & $a^{*}$ & $b^{*}$ \\
\hline RNP & 41.35 & 55.22 & 30.21 \\
S-RNP & 56.60 & 39.31 & 13.98 \\
Showed S-RNP & 45.68 & 50.33 & 21.41
\end{tabular}

${ }^{a}$ Note: $L^{*}$ is an indication of the perceived lightness/brightness, and $a^{*}$ and $b^{*}$ relate to the two opposing red-green and yellow-blue chroma axes, respectively. ${ }^{22}$

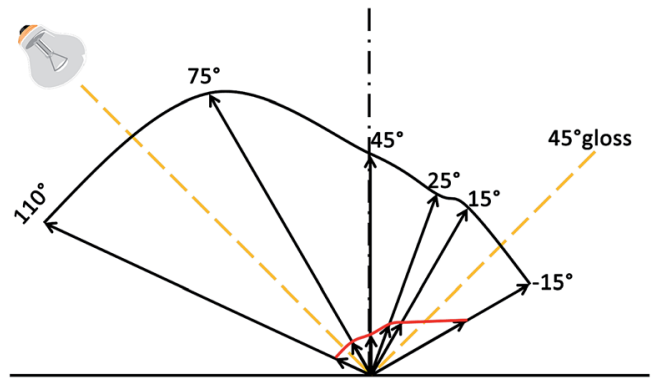

Fig. 5 The diffuse reflectance intensities at different angles for RNP (red curve) and S-RNP (black curve). The data are generated from Fig. 4.

dependent on the difference in the refractive index between the dispersed phase and the matrix. A significant difference in the refractive index results in strong scattering. Secondly, scattering is also related to the difference between the wavelength of light and the particle size. The optimal dispersed phase diameter for scattering light is about half of the wavelength of the light. Here, the light scattering of S-RNP is mainly caused by the air voids between $\mathrm{TiO}_{2}$ particles. After loading $\mathrm{TiO}_{2}$ particles on the paper, the difference in refractive index was not only that between cellulose (1.55) and air (1.00) but also partially between $\mathrm{TiO}_{2}$ (2.73) and air (1.00), ${ }^{19,20}$ leading to an increase in the difference in the refractive index. Consequently, the scattering of S-RNP evidently increased in comparison with that of RNP. Additionally, it is known from Mie theory that when the size of a particle is comparable to the wavelength of incident light, Mie scattering predominates, and the wavelength of light of Mie scattering is almost the same as the wavelength of the incident light. ${ }^{21}$ In other words, in the case of incident light of visible wavelength, Mie scattering can increase the white component in the reflected light. As a result, the fading of colored paper caused by the loaded $\mathrm{TiO}_{2}$ particles can be understood. In fact, it is very common for white particles with a higher refractive index to be used to obscure a background of contrasting color. ${ }^{19}$

\subsection{Effect of the showing mechanism on the morphology and color of paper loaded with $\mathrm{TiO}_{2}$}

3.2.1. Effect of the showing mechanism on the morphology of paper loaded with $\mathrm{TiO}_{2}$. According to the showing process mentioned in Experimental section 2.3, a trace amount of ionic liquid was introduced into the S-RNP sample to reduce scattering for showing the color. The morphology and components for the showed S-RNP were characterized by SEM and EDS mapping as shown in Fig. 6. Compared with the SEM image of the S-RNP sample (Fig. 3b), most of the air voids between $\mathrm{TiO}_{2}$ particles located on fibers are filled in the showed S-RNP (Fig. 6a). In addition, the EDS spectrum of the showed S-RNP shows that besides $\mathrm{C}, \mathrm{O}$, and $\mathrm{Ti}, \mathrm{N}, \mathrm{P}$, and $\mathrm{F}$ from [BMIm] $\mathrm{PF}_{6}$ are also found (Fig. 6b). Fig. 6c and d illustrate the distributions of $\mathrm{P}$ and $\mathrm{Ti}$, respectively. $\mathrm{P}$ homogeneously appears on the fibers of the paper, indicating that the air voids between the $\mathrm{TiO}_{2}$

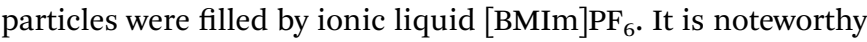
that the selection of this ionic liquid was based on the following 

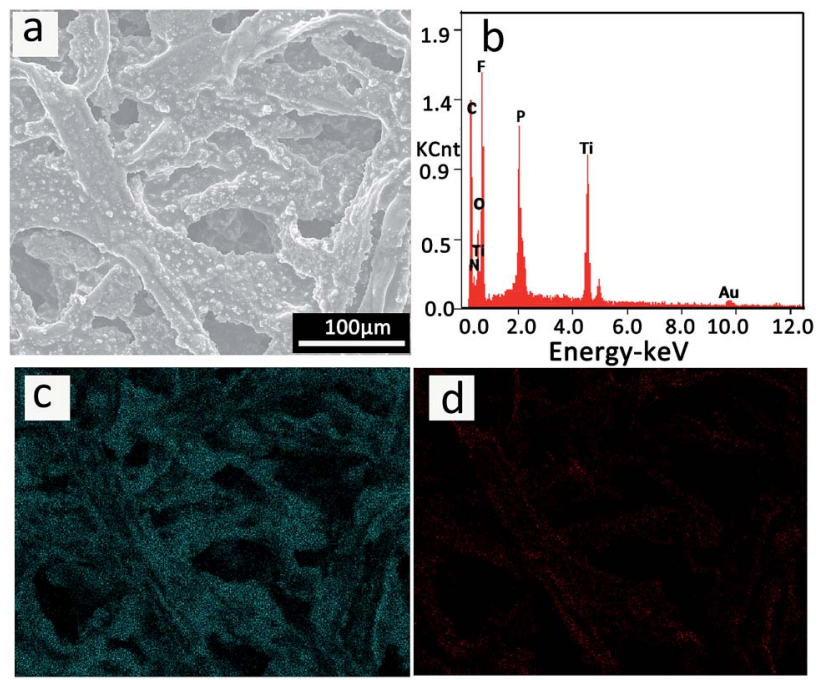

Fig. 6 (a) SEM image and (b) EDS spectrum of the showed S-RNP; (c) and (d) the corresponding elemental mapping of $\mathrm{P}$ and Ti, respectively.

considerations. Firstly, this ionic liquid possesses a high refractive index and low volatility as well as good affinity with the used paper. The high refractive index is beneficial for reducing scattering, the low volatility is favorable for the durable storage of the liquid in the scattering medium, and the good affinity is beneficial for decreasing the interface in the scattering medium.

3.2.2. Effect of the showing mechanism on the color of paper loaded with $\mathrm{TiO}_{2}$. The effect of the showing mechanism on the color of S-RNP was measured using a multi-angle spectrophotometer. The corresponding results, including color images and reflectance spectra in different directions, are shown in Fig. 7. In any direction, the reflectance of the showed S-RNP is lower than that of S-RNP, but slightly higher than that
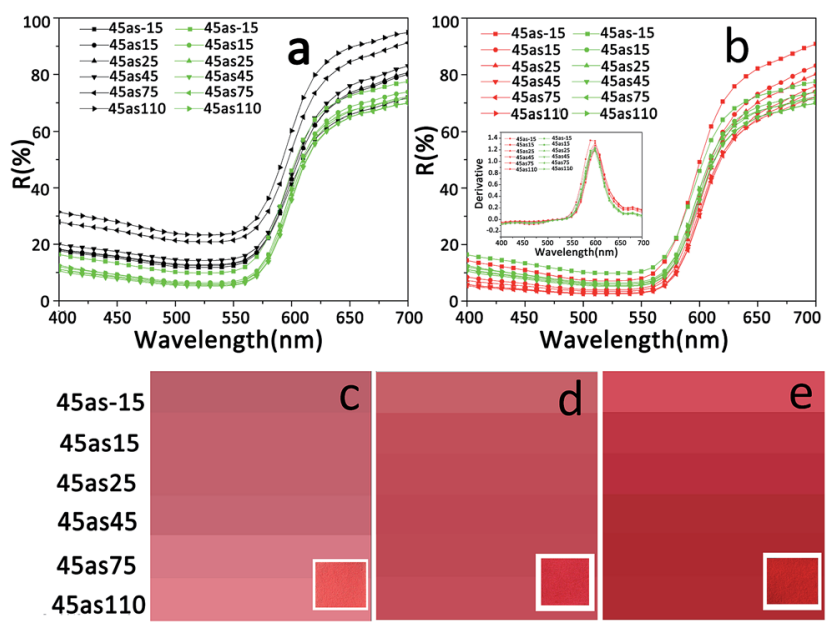

Fig.7 (a) and (b) Multi-angle reflectance spectra (black curves: S-RNP; green curves: the showed S-RNP; red curves: RNP). The inset in (b) shows the first derivative spectra (green curves: the showed S-RNP; red curves: RNP). (c), (d), and (e) Color images in different viewing directions (left: S-RNP; middle: the showed S-RNP; right: RNP). The insets in (c), (d), and (e) are photos of the corresponding samples. of RNP, indicating that the light absorption ability of the showed S-RNP is stronger than that of S-RNP, but slightly weaker than that of RNP (Fig. 7a and b). In other words, the color (red) of RNP fades when $\mathrm{TiO}_{2}$ particles are introduced into $\mathrm{RNP}$, while the introduction of a trace amount of ionic liquid into S-RNP can reproduce the original color of RNP to some extent. A similar principle has been reported previously. ${ }^{20}$ As mentioned already, the light scattering from the air voids between the $\mathrm{TiO}_{2}$ particles in S-RNP bleaches the color of S-RNP. As the air voids with a lower refractive index in S-RNP are filled with ionic liquid with a higher refractive index, the difference in refractive index between the dispersed phases and the matrix in the showed S-RNP is reduced. As a result, the scattering of the showed S-RNP is lower than that of S-RNP, and the color of the showed S-RNP is more visible than that of S-RNP. In principle, any colorless liquid with a higher refractive index can be selected to fill the scattering medium and reduce scattering. ${ }^{23-25}$ However, in consideration of stability in the showed color, a colorless stable non-volatile liquid is suitable to restore the bleached color originating from scattering. Moreover, a liquid with good affinity to the treated material should be chosen to eliminate interfaces in the scattering medium.

The color images shown in Fig. 7(c-e) illustrate that the color of the showed S-RNP is more obvious than that of S-RNP, and it is closer to that of RNP. In addition, the first derivative spectra shown in the inset of Fig. $7 \mathrm{~b}$ reveal that reflectance peaks at all directions of the showed S-RNP are similar to those of RNP and appear at a wavelength of $600 \mathrm{~nm}$, in the red color region, which indicates that the hue of the showed S-RNP is consistent with the original hue of RNP. The related values of $L^{*}, a^{*}$, and $b^{*}$ confirm the above results (Table 1 ).

The reflectance envelopes generated from Fig. 7 are shown in Fig. 8. Unlike S-RNP with distinct Mie scattering characteristics (Fig. 5), the profile of the reflectance intensities at different angles of the showed S-RNP is similar to that of RNP. The abovementioned change is attributed to the change in the morphology of the showed S-RNP. As shown in Fig. 6a, the air voids between $\mathrm{TiO}_{2}$ particles in the showed S-RNP almost disappear compared with those in S-RNP, as shown in Fig. 3b. It can be seen in Fig. $6 c$ and $d$ that the $P$ in the ionic liquid distributed uniformly around $\mathrm{Ti}$, indicating that the air voids between the $\mathrm{TiO}_{2}$ particles were filled with ionic liquid.

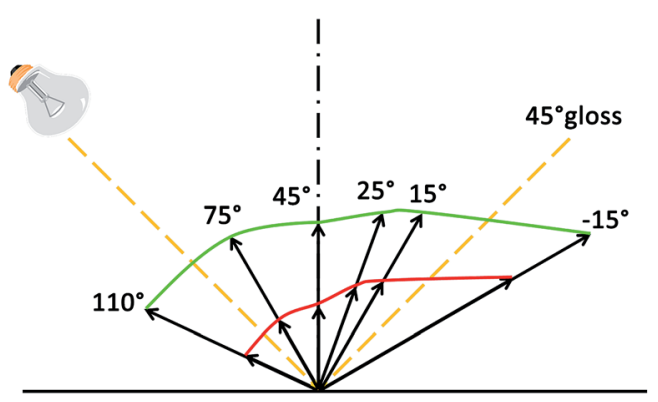

Fig. 8 The diffuse reflectance intensities at different angles for RNP (red curve) and the showed S-RNP (green curve). The data are generated from Fig. 7. 
Additionally, Fig. 8 also indicates that the characteristic of the specular reflectance of the showed S-RNP becomes more evident in comparison with S-RNP, which implies that the surface roughness of the showed S-RNP is reduced..$^{26}$ Evidently, the surface of the fibers in the showed S-RNP (Fig. 6a) becomes smoother than that of S-RNP (Fig. 3b). Although here the ionic liquid treatment is effective at showing faded color, a color difference still exists (as shown in Fig. 8, where the intensities at all angles of the showed S-RNP are higher compared with those of RNP). In fact, the scattering is related to many factors such as the size, shape, and optical properties of the dispersed phases as well as the medium. Therefore, it is difficult to completely eliminate the effect of scattering on the color only by filling the air voids with ionic liquid because all of the aforementioned factors cannot be completely recovered. Anyway, the faded SRNP can be distinctly restored by reducing light scattering through filling the air voids with liquid. In other words, filling air voids to reduce scattering is an efficient way to restore a faded painting.

\subsection{Effect of scattering on light transmission and absorption}

3.3.1. Effect of scattering on light transmission. Based on consideration of the following facts, the effect of scattering on the light transmission and absorption was determined. Scattering can reduce light transmission in the pigment layer, which results in color fading since the light absorption of pigments is decreased. ${ }^{19,20,27}$ In order to verify the above statement, the experiments described in Fig. 9 were performed. The air voids and cavities in the paper have light scattering properties. ${ }^{28}$ Therefore, the paper can be used as a scattering material. Here, 12 sheets of drinking paper with strong scattering were overlaid and compressed to obtain the paper sample with multi-layer scattering (MLS-DP). MLS-DP was placed upon print paper with fluorescence. The effect of scattering on light transmission could be probed by determining the fluorescence intensity in the case of MLS-DP with different numbers of layers. To determine the effect of the introduction of the ionic liquid on the optical path, a similar experiment was also performed where MLS-DP was replaced by MLS-DP filled with ionic liquid.

Fig. 10 presents the fluorescence spectra of MLS-DP (a) and the showed MLS-DP (b) sample with different numbers of layers placed upon the fluorescence paper. Clearly, for both samples, the fluorescence intensity at $440 \mathrm{~nm}$ decreases with the increase in the number of layers. However, for a given number of layers, the fluorescence intensity for the showed MLS-DP is higher than

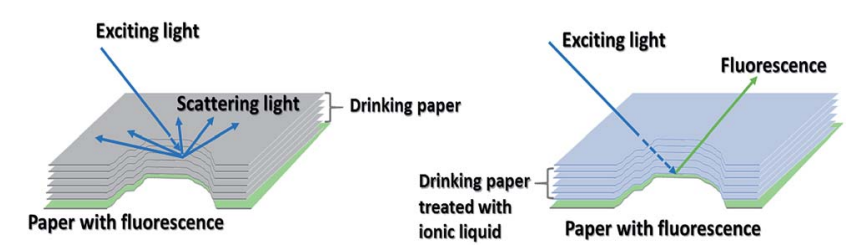

Fig. 9 Schematic diagram for fluorescence to detect the light path of incident light in a multi-layer scattering sample without (left) and with (right) filling with ionic liquid.
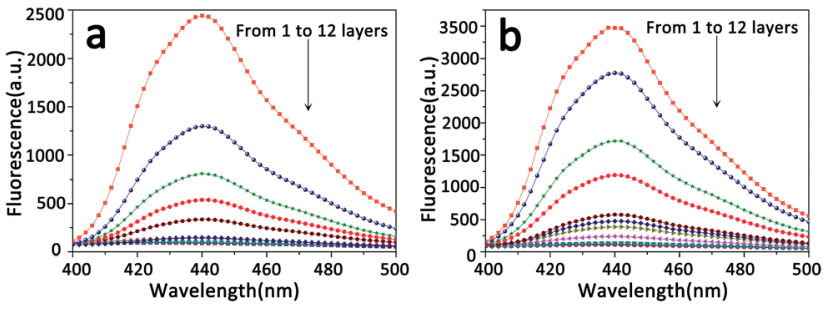

Fig. 10 Fluorescence spectra of the fluorescence paper placed under different numbers of layers of paper. (a) MLS-DP and (b) the showed MLS-DP.

that for MLS-DP, indicating that the excited light enters deeper into the showed MLS-DP than that into MLS-DP.

Fig. 11 more clearly illustrates the conclusions mentioned above. The fluorescence peak at $440 \mathrm{~nm}$ for MLS-DP and the showed MLS-DP as a function of the layer number is shown in Fig. 11. For both MLS-DP and the showed MLS-DP, the fluorescence intensity increases with the decrease of layer number, implying that the intensity of the exciting light penetrating into the samples increases with the decrease in the number of scattering layers. In other words, the scattering can reduce the optical path of incident light entering into the scattering medium. If some kind of pigment exists in this scattering medium, the light intensity absorbed by the pigment will be decreased. In this case, the color derived from the pigments must be weakened. Obviously, for a given number of layers, the fluorescence intensity for the showed MLS-DP is evidently greater than that for MLS-DP. These results firmly indicate that filling liquid into the air voids in a scattering medium is an efficient way to restore faded color paintings. It is worth noting that this method is limited to reducing scattering. As shown in Fig. 11, the fluorescence intensity is almost stable for both MLS-DP and the showed MLSDP when the number of layers exceeds 9. In fact, there is always scattering in a heterogeneous medium, and scattering increases with the thickness of the heterogeneous medium. Here, for the samples with 9 layers, the fluorescence intensity for MLS-DP is the same as that for the showed MLS-DP, implying that there is intrinsic scattering in MLS-DP. This intrinsic scattering is related to the optical characteristics of MLS-DP itself. However, reducing the thickness of the scattering layer is also an undoubtedly advisable method to reduce scattering. This protocol is very

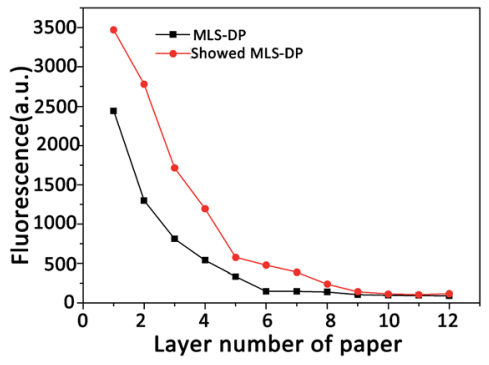

Fig. 11 Change in the maximum fluorescence intensity with different numbers of layers of paper. Black curve: MLS-DP; red curve: the showed MLS-DP. 
useful for application in the restoration of faded heritage relic surfaces covered with a scattering layer.

3.3.2. Effect of scattering on light absorption. In order to reveal the effect of scattering on the light absorption of the dye, a similar experiment, as described in the section on the effect of scattering on light transmission, was also carried out by replacing the fluorescence paper with red napkin paper (RNP). Fig. 12 illustrates the multi-angle reflectance spectra of RNP placed under MLS-DP (black curves) and the showed MLS-DP (green curves) with a different number of layers of paper as well as the corresponding color images. For MLS-DP with 12 and 8 layers, the reflectance spectra in any angle are nearly straight without any reflection peaks, indicating that the incident light is almost not absorbed by the bottom RNP. As shown in the corresponding color images in Fig. 12 (bottom), the color is almost white. The obvious absorption ranging from $400 \mathrm{~nm}$ to $550 \mathrm{~nm}$ occurs only when the number of layers is 1 , as shown in Fig. 12d (black curves). However, for the showed MLS-DP, the reflectance spectra indicate absorption even if the number of layers is 12 , and the absorption becomes more obvious with the decrease in the number of layers. These results are consistent with the corresponding color images shown in Fig. 12 (bottom). All the results mentioned above further confirm that the color of
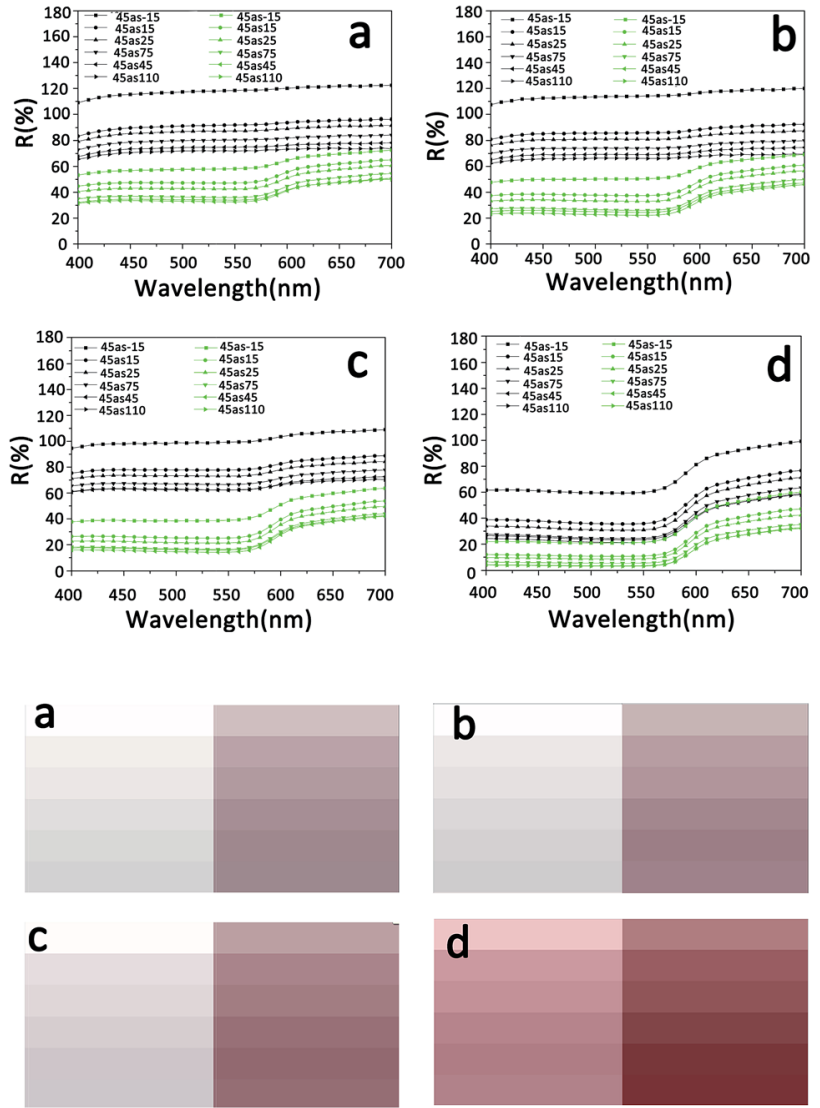

Fig. 12 (Top) Multi-angle reflectance spectra of RNP placed under different numbers of layers of paper ((a) 12 layers; (b) 8 layers; (c) 5 layers; (d) 1 layer). MLS-DP (black curves) and the showed MLS-DP (green curves). (Bottom) The corresponding color images. MLS-DP (left); the showed MLS-DP (right). the painting layer is bleached by the light path shortening caused by scattering, and the introduction of a little liquid into the scattering medium can restore the bleached color.

\subsection{A case of restoration of a faded ancient Chinese painting}

A faded ancient Chinese painting that was created on wooden building components as shown in Fig. 13a (Yuan Dynasty, 1316, Hancheng Puzhao Temple, Shaanxi, China), was restored using our proposed method mentioned above.

The colored drawings were on paper attached to wood boarding. As decorative paintings, they were mounted on the ceiling of a temple. The painting restored in this investigation is one of them. From the photograph of the painting shown in Fig. 13a, it can be seen that there are some contaminative white spots on the surface of the painting. These spots make the painting blurry and destroy the aesthetic perception of the painting. These spots adhere strongly on the painting, and are difficult to dissolve with solvents or to mechanically peel off from the extremely fragile painting. Therefore, it is hard to restore this painting with a traditional protocol. Based on careful analysis, the aforementioned method was applied to effectively solve this troublesome issue.

To investigate the spots on this ancient painting, the morphology on the micro-scale and the elemental components were determined. SEM images of pieces with spots are shown in
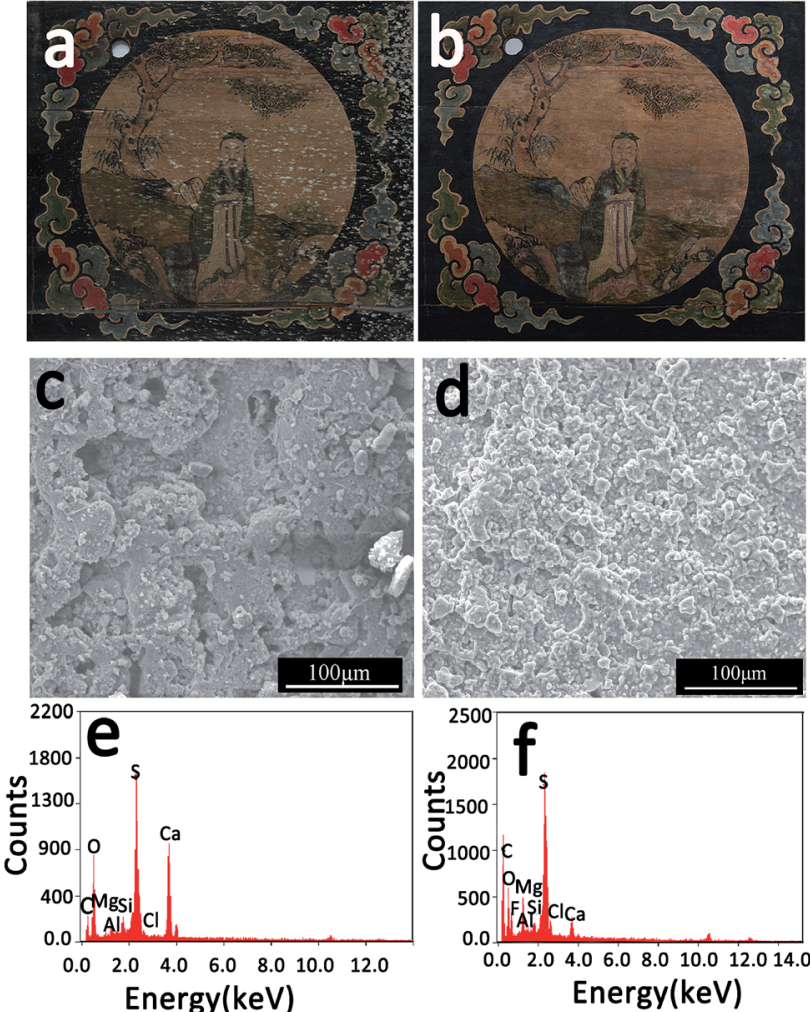

Fig. 13 Photographs ((a) original and (b) after treatment by ionic liquid) of the collected colored painting (Yuan Dynasty, 1316, Hancheng Puzhao Temple, Shaanxi, China); SEM images of the original painting (c) and the painting after treatment (d); EDS spectra (e) and (f) corresponding to (c) and (d), respectively. 
Fig. $13 \mathrm{c}$ and indicate that the spots consist of numerous small particles. Between the particles, there are a lot of air voids. This situation is similar to that mentioned above. Additionally, the element components of the spots shown in Fig. 13c and e imply that the spots are probably a white pigment composed of bentonite clay. The color covered by these spots may be shown if these air voids were filled by the ionic liquid to reduce scattering. Considering this, the areas with spots on the painting were treated by a trace amount of a mixture of acetone and $[\mathrm{BMIm}] \mathrm{PF}_{6}$ as used in the simulated investigation. The result is shown in Fig. 13b. As expected, after the treatment, even without the removal of the spots, the white spots are almost imperceptible but the original color completely appears! by comparing Fig. 13d and e, it can be found that the air voids of the treated area almost disappear. The appearance of fluorine in the EDS spectrum confirms that the air voids are filled by ionic liquid $[\mathrm{BMIm}] \mathrm{PF}_{6}$. These results are very similar to the simulation experiment results mentioned above. The actual restoration with satisfactory results confirms that reducing scattering through filling air voids with stable and non-volatile liquid is feasible for colour rendering of faded heritage relics.

\section{Conclusions}

Fading is very common in ancient color relics. Generally, in the protection field of cultural relics, fading is usually attributed to the degradation of pigments. In fact, the degradation of organic binding media is another reason for color fading, which leads to the formation of a lot of air voids in the continuous phase of a painting layer. Scattering originating from these air voids also causes the fading of the painting layer. In this investigation, a new point of view, the fading and showing mechanisms of ancient color relics based on light scattering induced by particles, was considered. From the experimental results, the following conclusions were obtained: (1) nanoparticles with a size comparable to the wavelength of incident light strongly blur the color of the painting layer though light scattering; (2) in principle, the scattering, which is caused by dust particles covered on the painting layer or by air voids originating from the degradation of the binding medium in the painting layer, would lead to blurred color; (3) filling air voids in the painting layer with a trace amount of non-volatile liquid would show the blurred color that resulted from scattering; and (4) it is worth noting that filling air voids in the painting layer to show blurred color is limited in the case of very strong scattering. In this situation, reducing the thickness of the scattering layer is an advisable method to reduce scattering. The aforementioned conclusions are very useful for application in the restoration of faded heritage relics.

\section{Conflicts of interest}

There are no conflicts to declare.

\section{Acknowledgements}

The research was financially supported by the Natural Science Foundation of China (21173140 and 20973105) and the
National Program on Key Basic Research Project of China (2012CB725302).

\section{References}

1 M. E. Kubik, Color. Des. Creativity, 2010, 5, 1-8.

2 R. M. Kabbani, Chem. Educ., 1997, 2, 1-18.

3 S. C. Pei and Y. M. Chiu, IEEE Trans. Image Process., 2006, 15, 3230-3234.

4 I. Bonaduce, C. Blaensdorf, P. Dietemann and P. C. Maria, J. Cult. Herit., 2008, 9, 103-108.

5 K. J. Hu, C. B. Bai, L. Y. Ma, K. Bai, D. B. Liu and B. B. Fang, Heritage Sci., 2013, 1, 1-14.

6 D. Hradil, T. Grygar, J. Hradilová and P. Bezdička, Appl. Clay Sci., 2003, 22, 223-236.

7 S. D. Smith, Applications in Plant Sciences, 2014, 2, 1-6.

8 J. H. Wei, D. B. Finkelstein, J. Brigham-grette, I. S. Castaneda and N. Nowaczyk, Sedimentology, 2014, 61, 1793-1811.

9 T. Cavaleri, A. Giovagnoli and M. Nervo, Procedia Chem., 2013, 8, 45-54.

10 J. Pfaender, S. M. Gray, I. P. Rich, S. Chapuis, R. K. Hadiaty and F. Herder, Hydrobiologia, 2014, 739, 155-161.

11 Y. Mizokami and M. A. Webster, J. Opt. Soc. Am. A, 2012, 29, A10-A18.

12 M. A. Rojas-Graü, A. Sobrino-López, M. S. Tapia and O. Martín-Belloso, J. Food Sci., 2006, 71, S59-S65.

13 C. S. McCamy, Color Res. Appl., 1993, 18, 3-10.

14 K. E. Torrance and E. M. Sparrow, J. Opt. Soc. Am., 1967, 57, 1105-1114.

15 J. J. Koenderink and A. J. Van Doorn, Int. J. Comput. Vis., 1999, 31, 129-144.

16 B. Van Ginneken, M. Stavridi and J. J. Koenderink, Appl. Opt., 1998, 37, 130-139.

17 M. Oren and S. K. Nayar, Int. J. Comput. Vis., 1995, 14, 227-251.

18 A. Aridgides, R. N. Pinnock and D. F. Collins, Am. J. Phys., 1976, 44, 244-247.

19 M. Juuti, K. Koivunen, M. Silvennoinen, H. Paulapuro and K. E. Peiponen, Colloids Surf., A, 2009, 352, 94-98.

20 T. Fabritius and R. Myllylä, J. Phys. D: Appl. Phys., 2006, 39, 2609-2612.

21 T. G. Deepak, G. S. Anjusree, S. Thomas, T. A. Arun, V. Shantikumar Nair and A. Sreekumaran Nair, RSC Adv., 2014, 4, 17615-17638.

22 I. L. Weatherall and B. D. Coonbs, J. Invest. Dermatol., 1992, 99, 468-473.

23 D. A. Zimnyakov and V. V. Tuchin, Quantum Electron., 2002, 32, 849-867.

24 T. Fabritius, E. Alarousu, T. Prykäri, J. Hast and R. Myllylä, Quantum Electron., 2006, 36, 181-187.

25 E. Alarousu, L. Krehut, T. Prykäri and R. Myllylä, Meas. Sci. Technol., 2005, 16, 1131-11377.

26 F. L. S. Cuppo, A. García-Valenzuela and J. A. Olivares, Color Res. Appl., 2013, 38, 177-187.

27 S. Kumar, N. K. Verma and M. L. Singla, Digest Journal of Nanomaterials and Biostructures, 2012, 7, 607-619.

28 H. L. Zhu, Z. Q. Fang, C. Preston, Y. Y. Li and L. B. Hu, Energy Environ. Sci., 2014, 7, 269-287. 